\title{
Erratum to: Sequential Monte Carlo filter based on multiple strategies for a scene specialization classifier
}

Houda Maâmatou ${ }^{1,2,3^{*}}$, Thierry Chateau', Sami Gazzah², Yann Goyat ${ }^{3}$ and Najoua Essoukri Ben Amara²

\section{Erratum}

In this version of this article that was originally published [1] there were errors in the titles of Figures 11 and 12.

The titles should be as follows:

Figure 11: Comparison of sample-proposal strategies. Pedestrian detection: a CUHK_Square dataset and b MIT traffic dataset. Car detection: c MIT traffic dataset and $\mathbf{d}$ Logiroad traffic dataset.

And

Figure 12: Comparison of both observation strategies. Pedestrian detection: a CUHK_Square dataset and b MIT traffic dataset. Car detection: c MIT traffic dataset and $\mathbf{d}$ Logiroad traffic dataset.

The original article has been revised.

The publisher apologises for these errors.

\section{Author details}

${ }^{1}$ Institut Pascal, Blaise Pascal University, 24 Avenue des Landais, Clermont-Ferrand, France. ${ }^{2}$ SAGE ENISo, University of Sousse, BP 264 Sousse Erriadh, Sousse, Tunisia. ${ }^{3}$ Logiroad, 2 Rue Robert Schuman, Nantes, France.

Received: 15 December 2016 Accepted: 15 December 2016 Published online: 17 January 2017

\section{Reference}

1. H Maâmatou, T Chateau, S Gazzah, Y Goyat, NEB Amara, Sequential Monte Carlo filter based on multiple strategies for a scene specialization classifier. EURASIP Journal on Image and Video Processing 2016, 40 (2016)

\footnotetext{
* Correspondence: houda.maamatou@etudiant.univ-bpclermont.fr ${ }^{1}$ Institut Pascal, Blaise Pascal University, 24 Avenue des Landais, Clermont-Ferrand, France

${ }^{2}$ SAGE ENISo, University of Sousse, BP 264 Sousse Erriadh, Sousse, Tunisia

Full list of author information is available at the end of the article
} 